Jerome F. O'Hara Jr MD, ${ }^{*}$ Marc I. Brand MD, $\dagger$ Azmy R. Boutros MD*

\title{
Acute airway obstruction following placement of a subclavian Hickman catheter
}

The purpose of this case report is to describe the events, intervention, and aetiology which led to acute airway obstruction in an adult patient afier the placement of a Hickman catheter. Airway obstruction secondary to superior vena cava obstruction occurred after placement of a subclavian vein Hickman catheter. This was felt to occur, in part, to a narrowed superior vena cava as evident by subclavian venography. It resulted in emergency oral tracheal intubation to relieve airway obstruction. Shortly after removal of the Hickman catheter, the signs of superior vena cava obstruction syndrome resolved and the patient was extubated without incidence. It is concluded that, although rare, the serious complication of acute airway obstruction can occur after placement of a Hickman catheter.

Cette observation a pour sujet la description des évènements, l'intervention et l'étiologie d'une obstruction aiguë des voies respiratoires chez un adulte après la mise en place d'un cathéter de Hickman. L'obstruction respiratoire secondaire à l'obstruction de la veine cave supérieure est survenue après la mise en place dans la veine sous-clavière d'un cathéter de Hickman. La vénographie sous-clavière montrait un rétrécissement de la veine cave supérieure. La trachée a été intubée en urgence pour lever l'obstruction des voies respiratoires. Peu de temps après avoir le retrait du cathéter, les signes d'obstruction de la veine cave sont disparus et le patient a été extubé sans incidents. En conclusion, une complication grave telle que l'obstruction aiguë des voies respiratoires peut survenir après la mise en place d'un cathéter de Hickman.

\section{Key words}

AIRWAY OBSTRUCTION; EQUIPMENT: Hickman catheter.

From the *Division of Anesthesiology, and $\dagger$ Department of General Surgery, The Cleveland Clinic Foundation, 9500

Euclid Avenue, Cleveland, Ohio 44195-5001.

Address correspondence to: Dr. Jerome F. O'Hara Jr. Accepted for publication 10th November, 1993.
The use of Hickman catheters has gained widespread acceptance since first described by Hickman, et al. ${ }^{1}$ in 1974. Although some patients selected for Hickman catheter placement have serious medical problems, the surgical procedure is usually uneventful and done under monitored anaesthetic care (MAC) with the infiltration of a local anaesthetic at the surgical field.

We report a case of an adult patient who had a right subclavian vein Hickman catheter placed, for the purpose of administering further chemotherapy, who developed an acute superior vena cava obstruction (SVCO) syndrome.

\section{Case report}

A 58-yr-old white woman, weighing $55 \mathrm{~kg}$, ASA physical status III, was diagnosed with acute myelogenous leukaemia (AML) six months before this admission. Her past medical history was significant for hypertension. Previously, a left subclavian Hickman catheter had been placed for chemotherapy. The patient gradually developed symptoms of left upper extremity, chest, and left neck swelling consistent with SVCO syndrome. When aspiration of blood through the catheter became difficult, it was removed. Two months later, the patient was scheduled for placement of a right subclavian Hickman catheter. On the morning of the scheduled surgery, bilateral subclavian venography with digital subtraction images revealed marked narrowing of the superior vena cava (SVC). Both subclavian veins were patent (Figure). She was in no acute distress and demonstrated no evidence of SVCO syndrome. Respiratory rate was $16 \mathrm{bpm}$, blood pressure was $160 / 95 \mathrm{mmHg}$, heart rate was regular at 80 beats per minute, and a baseline oxyhaemoglobin saturation $\left(\mathrm{SpO}_{2}\right)$ was $96 \%\left(\mathrm{FIO}_{2} 0.21\right)$.

The patient received midazolam, $2 \mathrm{mg} i v$ and a total of $150 \mu \mathrm{g}$ fentanyl $i v$ in the operating room. The surgical site was prepared and draped in a sterile fashion and the skin infiltrated with a $1: 1$ mix of lidocaine $1 \%$ and bupivacaine $0.25 \%$. The total volume of the local anaesthetic used was $20 \mathrm{ml}$ given in divided doses. The right 

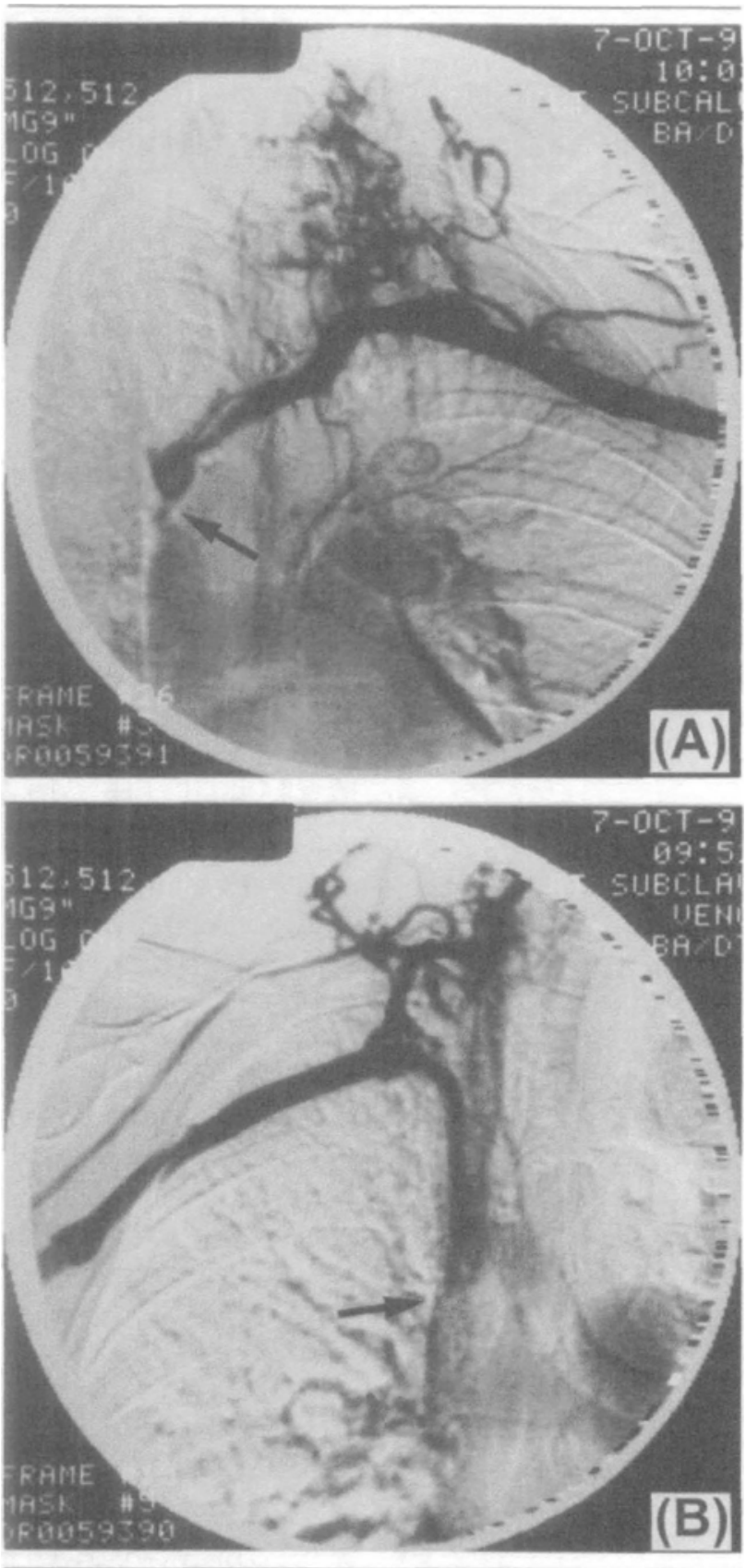

FIGURE Bilateral subclavian venography with digital subtraction images revealing marked narrowing of the superior vena cava (arrow). The radiographic study was performed on the morning of the scheduled surgery. I(A) left subclavian study, l(B) right subclavian study.

subclavian vein was cannulated on the first attempt and, with intermittent fluoroscopy, a guide wire was properly positioned into the SVC. A 12.5 French triple lumen catheter was then properly placed.

Twenty minutes after catheter placement when the surgical drapes were being removed, the patient began to complain of breathing difficulty. The $\mathrm{SpO}_{2}$ remained at
95-96\% $\left(\mathrm{FiO}_{2} 0.21\right)$ throughout. Bilateral breath sounds were present. A chest $x$-ray was rapidly obtained and ruled out a pneumothorax, widened mediastinum, or pleural effusion. Blood pressure remained between 130/ 70 to $170 / 90 \mathrm{mmHg}$ and the heart rate $70-80$ beats per minute. The patient began to exhibit progressively worsening inspiratory stridor and increased agitation.

Attempts to support ventilation by face mask were unsuccessful due to the patient's agitation. Thiopentone, 250 $\mathrm{mg}$, followed by succinylcholine, $40 \mathrm{mg}$, were given and a $7.0 \mathrm{~mm}$ oral endotracheal tube was placed without difficulty under direct laryngoscopy. Marked supraglottic oedema was noted. The presence of bilateral breath sounds and of an $\mathrm{PETCO}_{2}$ wave form confirmed proper placement of the endotracheal tube. The $\mathrm{SpO}_{2}$ was $99-100 \%\left(\mathrm{FIO}_{2} 1.0\right)$. The patient's breathing was controlled until muscle relaxant wore off and, 15 min later, she was awake and responding to commands. She breathed spontaneously with no difficulty. During this time the patient progressively developed upper extremity, neck, facial oedema and cyanosis. The catheter was promptly removed. Fifteen minutes later, marked improvement of the oedema and cyanosis was observed. The trachea was then extubated and the patient observed while in the operating room. She was calm and breathed normally. She was transported to the post-anaesthesia care unit and the clinical signs of upper torso oedema slowly dissipated. She remained there overnight. No further respiratory problems were encountered. The patient subsequently had a right saphenous vein placement of a triple lumen 12.5 French Davol catheter three days later under MAC and local anaesthesia without problems.

\section{Discussion}

We believe that this case describes the first report of an adult patient who developed an acute SVCO syndrome after placement of a right subclavian Hickman catheter into a narrowed, but patent SVC. Tracheal intubation for relief of airway obstruction was required. The aetiology was felt to be related to existing thrombus formation in the SVC and further mechanical intrinsic luminal occlusion that occurred after placement of a Hickman catheter. Previous case reports of patients with Hickman catheters and the diagnosis of SVCO syndrome have described the complication of airway obstruction. They include that of a 58-yr-old man who presented complaining of intense shortness of breath that was position related, ${ }^{2}$ a 60 -yr-old man after partial glossectomy and right radical neck dissection who had a left external jugular vein Hickman catheter placed and who required emergency tracheostomy. ${ }^{3} A$ third case involved a $14 \mathrm{mo}$ male infant who, shortly after tracheal extubation from an operation for placement of a Hickman catheter via 
right femoral vein, required emergency reintubation for worsening inspiratory stridor and airway obstruction.

Many benefits and a high degree of patient acceptance have made the Hickman catheter valuable in selected patients requiring continuous venous access. Indications for Hickman catheter placement include the ease of administering chemotherapy drugs, intravenous infusions, blood products, antibiotics, parenteral hyperalimentation, and in some patients, the need for extensive surveillance of serum haematological and blood chemistry variables.

Although these benefits exist, Hickman catheters are not without complications. The complications can be placed in one of two categories. First, acute complications are related to the surgical placement of catheter with the percutaneous technique. They include subclavian artery or aortic puncture or injury, innominate vein injury, misplacement of catheter, failure to cannulate the vein, air embolism, pneumothorax, haemothorax, and haemorrhage. Secondarily, post-placement complications arise after confirming proper placement during actual use. They include infection (most common), catheter malfunction, air embolism, haemorrhage, infiltration with extravasation of infusate, major thrombus formation, thromboembolism, SVCO syndrome, lymphatic leak, and catheter embolism. ${ }^{5,6}$

The most common diagnosis in a patient who develops a SVCO syndrome (without a Hickman catheter) is of a malignant tumour which applies extrinsic pressure occluding the SVC. A combination of symptoms such as a feeling of fullness, oedema of the head and neck, dyspnoea, headache, vertigo, decreased mentation, symptoms of laryngeal oedema, and airway compromise are common presenting features of patients who have a SVCO syndrome. $^{2}$ The onset of symptoms is usually subacute. One study noted that the average duration between the onset of symptoms and the diagnosis of SVCO syndrome secondary to a malignant tumour was 15 weeks. ${ }^{7}$

The circumstances are different if a patient has an indwelling SVC Hickman catheter and a diagnosis of a SVCO syndrome is made. The aetiology then is generally by intrinsic obstruction of the SVC secondary to thrombus formation. ${ }^{8}$ When one addresses the occurrence of a SVCO syndrome in a patient with an indwelling Hickman catheter, the incidence of thrombus formation must be discussed. Routine venography of asymptomatic patients reveals a high rate of thrombosis, varying from $4 \%$ to $42 \%{ }^{9}$

Signs and symptoms associated with a previous episode of superior vena cava thrombosis necessitate careful review of a preoperative venogram for subsequent catheter insertions. The presence of marked SVC narrowing in this setting suggests using a lower extremity venous access site. If cephalic cyanosis and swelling, laryngeal stridor, and acute respiratory distress occurs after Hickman catheter placement, it establishes the diagnosis of acute SVCO syndrome. Treatment is to secure the airway and remove the catheter.

In summary, we presented a case of a patient with a narrowed SVC who experienced acute airway obstruction secondary to the development of a SVCO syndrome and supraglottic oedema after placement of a Hickman catheter necessitating emergency tracheal intubation. The clinical signs of the SVCO syndrome reversed themselves after removal of the Hickman catheter allowing the patient to be safely extubated without incident. This case presents a rare, but serious, acute complication of the use of Hickman catheters.

\section{References}

1 Hickman RO, Buckner CD, Clift RA, Sanders JE, Stewart $P$, Thomas ED. A modified right atrial catheter for access to the venous system in marrow transplant recipients. Surg Gynecol Obstet 1979; 148: 871-5.

2 Kerr $H D$. Superior vena cava syndrome associated with a Hickman catheter. NY State J of Med 1990 April; 208-10.

3 Storper IS, Calcaterra TC. Laryngeal edema induced by neck dissection and catheter thrombosis. Am J of Otolaryngology 1992; 13: 101-4.

4 Black A, Lloyd-Thomas $A R$. An unusual case of stridor. Anaesthesia 1988; 43: 870-1.

5 Wagman $L D$, Neifeld JP. Experience with the Hickman catheter: unusual complications and suggestions for their prevention. J of Parenteral and Enteral Nutrition 1986; 10: 311-5.

6 Wisborg T, Flaatten H, Koller M-E. Percutaneous placement of permanent central venous catheters: experience with 200 catheters. Acta Anaesthesiol Scand 1991; 35: 49-51.

7 Schraufnagel DE, Hill $R$, Leech JA, Pare JAP. Superior vena cava obstruction. Is it a medical emergency? Am J Med 1981; 70: 1169-74.

8 Bertrand $M$, Presant. CA, Klein L, Scott E. Iatrogenic superior vena cava syndrome. A new entity. Cancer 1984; 54 : 376-8.

9 Haire WD, Lieberman RP, Edney J, et al. Hickman catheter-induced thoracic vein thrombosis. Cancer 1990; 66: $900-8$. 